\title{
Vigilance der Tierarzneimittel: Gemeldete unerwünschte Wirkungen im Jahr 2016
}

\author{
Müntener, Cedric R ; Kupper, Jacqueline ; Naegeli, Hanspeter ; Gassner, B
}

\begin{abstract}
A total of 253 reports of adverse reactions to veterinary medicinal products were received during the year 2016 representing a decrease of $13 \%$ compared to the previous year (292 reports). The majority of the reports described reactions affecting companion animals (178 dogs and 32 cats) as well as cattle (17 reports) and horses (10 reports). Most of the reactions reported were linked to the use of antiparasitics (145 reports), hormone products (26 reports) and antiinfectives (10 reports). 32 reports were generated from consultations with Tox Info Suisse in Zurich and involved mainly the excessive intake of flavoured tablets and, in some cases, the use of reconverted products (applied to another species than that authorized). Five signals were identified from the reports, which resulted in revisions of the product information in the sections addressing contraindications or adverse reactions.
\end{abstract}

DOI: https://doi.org/10.17236/sat00132

Other titles: Vigilance for veterinary medicinal products: Reports of adverse reactions in the year 2016

Posted at the Zurich Open Repository and Archive, University of Zurich

ZORA URL: https://doi.org/10.5167/uzh-144639

Journal Article

Published Version

Originally published at:

Müntener, Cedric R; Kupper, Jacqueline; Naegeli, Hanspeter; Gassner, B (2017). Vigilance der Tierarzneimittel: Gemeldete unerwünschte Wirkungen im Jahr 2016. Schweizer Archiv für Tierheilkunde, $159(11): 581-585$.

DOI: https://doi.org/10.17236/sat00132 


\title{
Vigilance der Tierarzneimittel: Gemeldete unerwünschte Wirkungen im Jahr 2016
}

\author{
C. R. Müntener1, 2, J. Kupper1,3, H. Naegeli1, B. Gassner² \\ ${ }^{1}$ Institut für Veterinärpharmakologie und -toxikologie der Universität Zürich, ${ }^{2}$ Swissmedic, Schweizerisches \\ Heilmittelinstitut, Bern, ${ }^{3}$ Tox Info Suisse, Zürich
}

\section{Zusammenfassung}

Im Jahr 2016 wurden 253 Meldungen zu unerwünschten Wirkungen von Tierarzneimitteln eingereicht, was eine Abnahme von 13\% gegenüber dem Vorjahr (292 Meldungen) bedeutet. Am häufigsten wurden Reaktionen bei Kleintieren (178 Hunden und 32 Katzen) sowie bei Rindern/Kälbern (17 Meldungen) und Pferden (10 Meldungen) eingereicht. Die am häufigsten betroffenen Medikamentengruppen waren Antiparasitika (145 Meldungen), Hormonpräparate (26) und Antiinfektiva (10). 32 Fälle, die von Tox Info Suisse übermittelt wurden, betrafen mehrheitlich die Aufnahme von Tierarzneimitteln mit Aromastoffen in Überdosis sowie einige Umwidmungen. Aus den gesammelten Meldungen wurden 5 Signale identifiziert, welche zur Anpassung der Arzneimittelinformation der betroffenen Präparate in den Rubriken „unerwünschte Wirkungen“ oder „Kontraindikationen" führten.

Schlüsselwörter: Pharmacovigilance, Antiparasitika, Gentamicin, Imidacloprid, Eisendextran

\section{Vigilance for veterinary medicinal products: Reports of adverse reactions in the year 2016}

A total of 253 reports of adverse reactions to veterinary medicinal products were received during the year 2016 representing a decrease of $13 \%$ compared to the previous year (292 reports). The majority of the reports described reactions affecting companion animals (178 dogs and 32 cats) as well as cattle (17 reports) and horses (10 reports). Most of the reactions reported were linked to the use of antiparasitics (145 reports), hormone products (26 reports) and antiinfectives (10 reports). 32 reports were generated from consultations with Tox Info Suisse in Zürich and involved mainly the excessive intake of flavoured tablets and, in some cases, the use of reconverted products (applied to another species than that authorized). Five signals were identified from the reports, which resulted in revisions of the product information in the sections addressing contraindications or adverse reactions.

Keywords: pharmacovigilance, antiparasitics, gentamicin, imidacloprid, iron dextran

\section{Einleitung}

Vor der Zulassung eines Tierarzneimittels müssen klinische Studien durchgeführt werden, die sich aus Zeit- und Kostengründen auf eine limitierte Anzahl Tiere beschränken. Für die Aufdeckung sehr seltener Ereignisse wie unerwünschte Wirkungen oder Interaktionen, die mit einer Frequenz von höchstens 1:10‘000 auftreten (Swissmedic, 2016), ist die Anzahl untersuchter Tiere deshalb oft nicht ausreichend. Damit die Sicherheit der Patienten trotzdem gewährleistet wird, müssen spontan gemeldete Reaktionen während der ganzen „Lebensdauer“ eines Heilmittels gesammelt und ausgewertet werden. Diese Auswertungen finden in der Schweiz sowohl bei Swissmedic als auch beim Institut für Virologie und Immunologie (IVI, Mittelhäusern) statt. Tierärzte und Apotheker, die Heilmittel im Rahmen ihres Berufs anwenden oder abgeben, sind gesetzlich verpflichtet, unbekannte oder schwerwiegende unerwünschte Wirkungen zu melden (Verordnung über die Arzneimittel Art. 35 Abs. 1). Für Drittpersonen besteht eine freiwillige Möglichkeit, die beobachteten Ereignisse zu melden (Heilmittelgesetz Art. 59 Abs. 4). Als Meldestellen stehen entweder die zuständigen Behörden (Swissmedic, IVI) oder ein peripheres Meldezentrum im Institut für Veterinärpharmakologie und -toxikologie zur Verfügung. Die Auswertung der letzten Jahre zeigt, dass Tierbesitzer sich in der Mehrheit zunächst an die behandelnden Tierärzte wenden und diese anschliessend die https://doi.org/ $10.17236 /$ sat00132

Eingereicht: 13.07.2017 Angenommen: 30.08 .2017 
Vigilance der Tierarzneimittel: Gemeldete unerwünschte Wirkungen im Jahr 2016

C.R. Müntener et al.
Vertriebsfirmen informieren. Gemäss ihrer gesetzlichen Verpflichtung leiten die Letztgenannten dann die Informationen an die zuständigen Behörden weiter. Alternativ können sich Tierbesitzer, insbesondere bei irrtümlichen Anwendungen oder Aufnahme von Tabletten in Überdosis durch ihre Tiere an Tox Info Suisse wenden. Diese Stelle leitet alle Beratungsfälle, bei welchen sowohl ein Tier als auch ein Tierarzneimittel involviert sind, periodisch an Swissmedic weiter.

Im Zentrum eines Tierarzneimittelvigilance-Systems steht die Evaluation der Meldungen nach international anerkannten Kriterien (ABON-System; EMEA, 2003a), mit dem Ziel, bisher unbekannte Risiken oder Änderungen der Eigenschaften bekannter Risiken zu entdecken und die Sicherheit der Tierarzneimittel sowohl für behandelte Tiere als auch für Tierärzte und Besitzer laufend zu verbessern. Mögliche Konsequenzen dieser Evaluation sind Modifikationen der Arzneimittelinformation in den Rubriken „Unerwünschte Wirkungen“, „Vorsichtsmassnahmen“ oder „Kontraindikationen“ (Müntener et al., 2004). Bei Tierarzneimitteln mit einer Zulassung zur Anwendung bei Nutztieren spielt der Schutz der Konsumenten vor unerwünschten Lebensmittelrückständen eine wichtige Rolle. In solchen Fällen können Meldungen zu unerwünschten Rückständen in Fleisch, Eier, Honig oder in der Milch zu Anpassungen der Rubrik „Absetzfristen“ führen. Für die Sicherheit der Impfstoffe und eventuell notwendige Anpassungen ihrer Arzneimittelinformation ist in der Schweiz die Vaccinovigilance Stelle des IVI zuständig (Albrecht und Ottiger, 2016).

Wir präsentieren nachfolgend eine Übersicht der im Jahr 2016 bei Swissmedic eingegangenen Meldungen zu Tierarzneimitteln mit Auswertungen in Bezug auf betroffene Tierarten, Medikamentenklassen und hinsichtlich Kausalzusammenhang zwischen Anwendung und unerwünschter Reaktion. Einige Beispiele werden summarisch präsentiert.

\section{Meldungen zu zugelassenen Tierarzneimitteln}

Im Jahr 2016 wurde mit einem Total von 253 Meldungen eine Abnahme von 13\% gegenüber dem Jahr 2015 verzeichnet (292 Meldungen). Die Verteilung der Quellen entspricht den früheren Jahren: 72.3\% der Meldungen $(\mathrm{N}=183)$ wurden von Vertriebsfirmen eingereicht, 13.4\% von praktizierenden Tierärzten $(\mathrm{N}=34)$ und $12.6 \%$ $(\mathrm{N}=32)$ wurden von Tox Info Suisse im Rahmen von Beratungen aufgenommen. Die dominierende Position der Industrie als Hauptquelle von Meldungen wird auch in Nachbachländern verzeichnet: In einer Publikation zu den Jahren 2011 bis 2013 stammte die überwiegende Mehrheit der Meldungen in Deutschland aus der Phar- maindustrie mit leicht sinkendem Anteil von Direktmeldungen durch Praktiker (Palm et al., 2014). In der Schweiz wurden die restlichen Meldungen an Swissmedic entweder direkt von Tierbesitzern (1.2\% oder $\mathrm{N}=3$ ) oder von einem Amt $(0.4 \%, N=1)$ eingereicht. Bei den betroffenen Tierarten sind nach wie vor die Kleintiere in der Überzahl, wobei 178 Meldungen (70.4\%) zu unerwünschten Wirkungen bei Hunden und 32 (12.6\%) bei Katzen verzeichnet werden konnten. Ein Kleintier-Anteil von ca. $80 \%$ bei den Meldungen wird auch durch ausländische Systeme beobachtet: in Grossbritannien wurden im Jahr 2015 bei den Kleintieren 4'329 (76.8\%) von total 5'638 Meldungen von Reaktionen verbucht (Cooles et al., 2017). Eine steigende Tendenz zugunsten der Kleintiere wurde in dieser Publikation auch erwähnt. In abnehmender Reihenfolge betrafen die restlichen Meldungen von 2016 aus der Schweiz Rinder und Kälber (17 Meldungen) sowie Pferde (10 Meldungen). Für alle anderen Zieltierarten gingen während des ganzen Jahres weniger als 5 Meldungen ein. Hingegen gingen keine Meldungen zu Reaktionen bei Anwendern oder Tierbesitzern ein.

Tabelle 1 präsentiert die eingegangenen Meldungen, sortiert nach Arzneimittelklassen gemäss dem ATCvet Code. Am häufigsten wurden, wie in früheren Jahren, unerwünschte Wirkungen nach der Anwendung von Antiparasitika gemeldet (145 Meldungen, 57.2\%; Müntener et al., 2016). Danach folgten Hormonpräparate (26 Meldungen, 10.3\%), Antiinfektiva (19 Meldungen, 7.5\%) und Präparate zur Behandlung des muskulo-skelettalen Systems (17 Meldungen, 6.7\%, meistens als nicht-steroidale Entzündungshemmer). Für alle anderen Präparategruppen lagen weniger als 15 Meldungen ( $5 \%$ des Totals) für das ganze Jahr vor. 39 Meldungen zu Antiparasitika betrafen eine vermutete Unwirksamkeit. Am häufigsten wurden Halsbänder erwähnt, aber auch Präparate in Form von Spot-On oder Tabletten zum Einnehmen. Grösstenteils wurde eine Unwirksamkeit gegen Zecken vermutet, da Tierbesitzer trotz der Applikation der Präparate Zecken auf ihrem Tier entdeckten. Auf diese Problematik wurde bereits früher eingegangen (Müntener et al., 2016): Es muss grundsätzlich bedacht werden, dass kein Tierarzneimittel eine 100-prozentige Wirkung beanspruchen kann und dass die Richtlinie der Europäischen Heilmittelbehörde zur Zulassung solcher Präparate auch eine Wirksamkeit attestiert, wenn in den ersten 24 bis 48 Stunden nach Applikation einzelne lebende Zecken auf dem Tier gefunden werden (EMA, 2007). Die meisten dieser Meldungen wurden mit der Beurteilung „möglich“ oder „zu wenig Informationen“ abgelegt, insbesondere wenn die Latenz zwischen der Applikation und der Beobachtung von Zecken auf dem Tier unbekannt war. In derselben Gruppe der Antiparasitika erhielten wir 42 Meldungen zu einem Präparat mit dem neuen, bei Hunden gegen Flöhe und Zecken oral einsetzbaren Wirkstoff Fluralaner. Die relativ hohe Anzahl der Meldungen (16.6\% des Jahresto- 
Tabelle 1: Aufteilung der im Jahr 2016 gemeldeten unerwünschten Wirkungen, sortiert nach ATCvet Code, mit spezifischer Darstellung für Hunde und Katzen. Der fiktive Code QZ erlaubt Meldungen über unerwünschte Arzneimittelwirkungen bei umgewidmeten Präparaten (d.h. nicht bei der zugelassenen Tierart und/oder Indikation angewendet) spezifisch zu gruppieren.

\begin{tabular}{|c|c|c|c|}
\hline \multirow[t]{2}{*}{ Medikamentengruppe nach ATCvet } & \multicolumn{3}{|c|}{ Anzahl Meldungen (\% des jeweiligen Totals) } \\
\hline & Alle Spezies & Hund & Katze \\
\hline QA: Gastrointestinaltrakt & $4(1.6 \%)$ & $2(1.1 \%)$ & 0 \\
\hline QB: Blut und blutbildende Organe & $5(2 \%)$ & 0 & 0 \\
\hline QC: Kardiovaskuläres System & $13(5.1 \%)$ & $12(6.7 \%)$ & $1(3.1 \%)$ \\
\hline QG: Urogenitalsystem, Geschlechtshormone & $2(0.8 \%)$ & $1(0.6 \%)$ & 0 \\
\hline QH: Hormonpräparate (ausser Sexualhormone und Insulinderivate) & $26(10.3 \%)$ & $21(11.8 \%)$ & $4(12.5 \%)$ \\
\hline QJ: Antiinfektiva & $19(7.5 \%)$ & $5(2.8 \%)$ & 0 \\
\hline QL: Antineoplastische und immunmodulierende Präparate & $3(1.2 \%)$ & $3(1.7 \%)$ & 0 \\
\hline QM: Muskel- und Skelettsystem & $17(6.7 \%)$ & $13(7.3 \%)$ & $2(6.3 \%)$ \\
\hline QN: Nervensystem & $5(2 \%)$ & $2(1.1 \%)$ & $2(6.3 \%)$ \\
\hline QP: Antiparasitika & $145(57.2 \%)$ & $114(64.1 \%)$ & $20(62.4 \%)$ \\
\hline QR: Respirationstrakt & $1(0.4 \%)$ & 0 & 0 \\
\hline QS: Sinnesorgane & $5(2 \%)$ & $5(2.8 \%)$ & 0 \\
\hline "QZ": Umgewidmete Präparate & $8(3.2 \%)$ & 0 & $3(9.4 \%)$ \\
\hline Total & 253 & 178 & 32 \\
\hline
\end{tabular}

tals) lässt sich primär durch die Markteinführung dieses Präparates erklären. In einer 1984 publizierten Studie beschrieb Weber einen inzwischen nach ihm benannten Effekt, bei welchem die neue Zulassung eines Heilmittels zu einer Spitze von Meldungen in den ersten zwei Jahren der Vermarktung führt (Weber, 1984). Die für das erwähnte Präparat gemeldeten Symptome waren Durchfall, Erbrechen, Inappetenz, Anorexie und Lethargie. Bis auf die Letztgenannten sind alle Symptome in der Arzneimittelinformation des Präparates erwähnt. In Einzelfällen wurde auch über Ataxie, Alopezie oder Hepatopathie berichtet. Diese Symptome konnten nach der Evaluation nicht eindeutig zugeordnet werden. Bei einer Zunahme solcher Meldungen müsste das Sicherheitsprofil des Präparates erneut evaluiert und die Arzneimittelinformation eventuell angepasst werden.

Vier Meldungen zu Antiinfektiva betrafen positive Rückstände nach eingehaltener Absetzfrist. Bei 3 Fällen gab es für eine definitive Beurteilung nicht genügend Informationen. Beim vierten Fall lag eine off-label Anwendung vor (die Kuh wurde doppelt so lange wie zugelassen therapiert) und es konnte keine Kausalität etabliert werden.

Insgesamt erhielten wir 8 Meldungen zu umgewidmeten Präparaten, die bei einer anderen als der zugelassenen Tierart eingesetzt wurde. Davon betrafen 2 Fälle die Anwendung von Permethrin-haltigen Spot-On Präparaten bei Katzen. Diese Problematik wurde in der Fachliteratur bereits mehrfach erwähnt (Müntener et al., 2013; Boland und Angles, 2010), neu werden auch intravenöse Lipidemulsionen zur Behandlung solcher Vergiftungen empfohlen (Peacock et al., 2015). Die zwei irrtümlich behandelten Tiere erholten sich glücklicherweise vollständig. Die anderen Meldungen betrafen Kaninchen, Meerschwein- chen, Frettchen oder Kanarienvögel. Im letzten Fall erhielten wir die Meldung einer Anwendung von einem für Hunde zugelassenem Präparat mit Imidacloprid. $26 \mathrm{Ka}$ narienvögel wurden vom Züchter mit je einem Tropfen des Produkts behandelt. Sieben Vögel waren danach apathisch, einer starb. Das eingesetzte Präparat ist ein Insektizid zur äusseren Anwendung bei Hunden und enthält unter anderem Imidacloprid einen Wirkstoff, welcher für gewisse Vogelarten besonders toxisch ist. In der Fachliteratur finden sich Angaben zu einer LD50 zwischen 25 und $50 \mathrm{mg} / \mathrm{kg}$ bei Kanarienvögeln (Gibbons et al., 2015). Die im verwendeten Präparat enthaltenen 100 mg Imidacloprid pro $\mathrm{ml}$ entsprechen ca. $5 \mathrm{mg}$ pro Tropfen. Bei einem in der Meldung angegebenen Durchschnittskörpergewicht von $7 \mathrm{~g}$ erhielten die Vögel somit ein Dosisäquivalent von $714 \mathrm{mg} / \mathrm{kg}$, das heisst bis zu $14 \mathrm{mal}$ die LD50-Dosis. Diese Meldung wurde mit der Kausalität „möglich“ abgelegt und die Arzneimittelinformation wurde mit der neuen Kontraindikation „Nicht bei Kanarienvögeln anwenden“ ergänzt (Tierarzneimittelkompendium der Schweiz, 2017).

Im Oktober 2016 erhielten wir Meldungen zu unerwünschten Wirkungen nach intravenöser Anwendung von Gentamicin-Lösungen bei Pferden. Mindestens 6 Pferde reagierten mit „Hyperhidrosis, Unruhe, Tachykardie, Zittern/Muskeltremor, Urtikaria“, Kolik-ähnliche Symptome und, in einem Fall, Zusammenbruch. Nach einer anfänglichen Vermutung, die höhere Dosierung von $6.6 \mathrm{mg} / \mathrm{kg}$ könnte für die Symptome verantwortlich sein, wurde ein Qualitätsproblem vermutet. Die relativ hohe Dosis ist zwar für die in der Schweiz zugelassenen Präparate eine off-label Anwendung, entspricht aber den Empfehlungen der Arzneimittelinformationen der in der EU zugelassenen Präparate. In derselben Zeit wurden sowohl 
Vigilance der Tierarzneimittel: Gemeldete unerwünschte Wirkungen im Jahr 2016

C.R. Müntener et al. in Grossbritannien als auch in Deutschland ähnliche Reaktionen beobachtet und bestimmte Chargen von Präparaten in diesen zwei Ländern vom Markt genommen. Seit Anfang des Jahres 2017 wurden, nach Wechsel des Präparates, keine unerwünschten Reaktionen mehr aus der Schweiz gemeldet. Die Fälle konnten bisher nicht abschliessend beurteilt werden, dennoch bleibt eine Kontamination des Wirkstoffs Gentamicin (nicht aber des Fertigpräparates) eine plausible Hypothese.

Schliesslich erreichten uns im Jahr 2016 fünf Meldungen von Reaktionen nach Anwendung von 3 verschiedenen Präparaten mit Eisendextran. Es waren sowohl Kälber (2 Meldungen, 3 Tiere) als auch Schweine (3 Meldungen, 43 Tiere) betroffen. Die Symptome waren in verschiedenen Kombinationen „pumpende Atmung, Kreislaufschock, Speicheln, Zyanose, Torkeln, Umfallen/Kollaps, Festliegen“(Originalwortlaut der Meldungen). Die parenterale Injektion von Eisenverbindungen kann bei jungen Tieren, insbesondere bei Vitamin E und Selenmangel, zu allergischen Reaktionen führen (Kroker, 2010). Im Laufe der letzten 10 Jahre wurden uns 15 solcher Meldungen eingereicht. Diese unerwünschten Wirkungen sind nun in den Arzneimittelinformationen aller betroffenen Präparate erwähnt (Tierarzneimittelkompendium der Schweiz, 2017). Alle 5 Meldungen wurden mit der Kausalität „möglich“ versehen und die Texte der Rubrik „unerwünschte Wirkungen“ wurden für alle Präparate auf denselben Stand gebracht.

Im Jahr 2016 wurden insgesamt 5 Signale identifiziert, 3 aus periodischen Berichten zur Sicherheit, eines aus eingereichten Meldungen und eines aus einer anderen Quelle. Alle Signale führten zu einer Anpassung der Rubriken „unerwünschte Wirkungen“ oder „Kontraindikationen“.

\section{Beratungen von Tox Info Suisse}

Im Jahr 2016 führte Tox Info Suisse 39'543 Beratungen durch, wovon in 2'002 Fällen Tiere betroffen waren. Die am häufigsten diskutierten Fälle betrafen absichtliche oder versehentliche Expositionen von Tieren mit diversen Noxen, Human- oder Tierarzneimitteln. Entsprechend einer langjährigen Vereinbarung mit Swissmedic werden alle Fälle, bei welchen sowohl Tiere als auch Tierarzneimittel involviert sind, periodisch weitergeleitet. $2016 \mathrm{gab}$ es 80 solcher Fälle. Die Verteilung der Medikamentenklassen dieser Fälle entspricht derjenigen in früheren Jahren: Antiparasitika waren mit $36.1 \%$ am häufigsten involviert, gefolgt von Entzündungshemmern (25.5\%) und Antiinfektiva (12.1\%). 40\% der Fälle ( $\mathrm{N}=32$ ) wiesen Minimalkriterien für eine Aufnahme ins System auf: Es waren genügend Informationen vorhanden und die Anwendung des Tierarzneimittels war absichtlich oder es lag eine gezielte Suche und Aufnahme durch das Tier aufgrund von intrinsischen Charakteristika wie z.B. Aromastoffen, vor. In 17 Fällen wurde die akzidentelle Aufnahme von Tabletten, meistens in Überdosis, durch 15 Hunde und 2 Katzen beschrieben. Am häufigsten (10 Fälle, 58.8\% der akzidentellen Aufnahmen) waren Entzündungshemmer involviert: die grösste Menge wurde bei einem 6 jährigen Appenzeller-Hund mit einem Körpergewicht von $25 \mathrm{~kg}$ beschrieben, welcher 80 Tabletten mit je $100 \mathrm{mg}$ Carprofen aufnahm. Die Gesamtmenge von $8 \mathrm{~g}$ war äquivalent mit einer 57 -fachen Überdosierung $(228 \mathrm{mg} / \mathrm{kg})$ und führte zur Apathie und Appetitlosigkeit. Für diesen Fall lagen keine weiteren Informationen zum Ausgang vor. Weitere Fälle beschrieben die Aufnahme von Antiparasitika (3 Fälle), ACE-Hemmern (2 Fälle) oder Antibiotika (2 Fälle). In den meisten Fällen waren die Tiere zur Zeit der Beratungen asymptomatisch oder hatten die Tabletten bereits erbrochen, so dass nur selten Zusatzinformationen zum weiteren Verlauf erhalten werden konnten. Alle Fälle beschreiben eine unerwünschte Nebenwirkung der Zugabe von Aromastoffen in Tabletten: Zwar ist die freiwillige Aufnahme durch die Tiere verbessert, aber gleichzeitig besteht die Gefahr einer akzidentellen Überdosierung, da durch die Assoziation mit einer Belohnung gezielt danach gesucht wird. Neuzugelassene oral applizierte Präparate, die Aromastoffe enthalten, werden deshalb systematisch mit einem entsprechenden Warnhinweis versehen (Tierarzneimittelkompendium der Schweiz, 2017).

\section{Schlussfolgerung}

Im Jahr 2016 wurde erstmals eine Abnahme der eingereichten Meldungen verzeichnet. Auf Grund der Natur eines Spontanmeldesystems kann dies nicht auf eine Änderung im Sicherheitsprofil eines oder mehrerer zugelassenen Präparate zurückgeführt werden. Zudem war in den Meldungen ein neues Präparat überdurchschnittlich vertreten. Deshalb muss diese Abnahme im Rahmen der normalen Schwankungen betrachtet werden. Die Pharmacovigilance für Tierarzneimittel bleibt ein wichtiges Werkzeug zur Verbesserung der Anwendungssicherheit und jede erstattete Meldung kann entscheidend dazu beitragen.

\section{Dank}

Wir möchten uns bei allen Personen bedanken, die sich im Laufe des Jahres die Zeit nahmen, Meldungen zu erstatten oder sich an der Evaluation der Meldungen beteiligten. 


\section{Pharmacovigilance vétérinaire: effets indésirables annoncés en 2016}

En 2016, 253 annonces d'effets indésirables de médicaments vétérinaires ont été enregistrées, ce qui représente une diminution de 13\% par rapport à l'année précédente (292 annonces). Ce sont principalement des annonces concernant des réactions chez les petits animaux (178 chiens et 32 chats) ainsi que chez les bovins (17) et les chevaux(10) qui ont été faites. Les groupes de médicaments les plus souvent impliqués étaient les antiparasitaires (145 annonces), les préparations hormonales (26) et les anti-infectieux (10). 32 cas transmis par Tox Info Suisse, concernaient principalement la prise en surdose de médicaments aromatisés ainsi que quelques reconversions. Cinq signaux issus de l'ensemble de ces annonces ont été identifiés, qui ont conduit à une adaptation des informations des produits concernés dans les rubriques „effets indésirables“ ou „contre-indications“.

\section{Literatur}

Albrecht N., Ottiger HP.: Vaccinovigilance Schweiz: Gemeldete unerwünschte Wirkungen während der letzten 13 Jahre. Schweiz. Arch. Tierheilk. 2016, 158: 251-258.

Boland LA., Angles JM.: Feline permethrin toxicity: Retrospective study of 42 cases. J. Feline Med. Surg. 2010, 12: 61-71.

Cooles S., Diesel G., Blenkinsop J.: Suspected adverse events, 2015. Vet. Rec. 2017, 180: 467-469.

Gibbons D., Morrissey C., Mineau P.: A review of the direct and indirect effects of neonicotinoids and fipronil on vertebrate wildlife. Environ. Sci. Pollut. Res. Int. 2015, 22: 103-118.

Kroker R.: Vitamine und Spurenelemente. In: Pharmakothe rapie bei Haus- und Nutztieren. Eds. W. Löscher, FR. Ungemach und R. Kroker, Enke Verlag, Stuttgart, 2010, 363-374.

Müntener C., Gassner B., Demuth D. C., Althaus F. R., Zwahlen R.: Pharmacovigilance für Tierarzneimittel in der Schweiz. Schweiz. Arch. Tierheilk. 2004, 146: 499-505.

Müntener C., Bruckner L., Kupper J., Althaus FR., Schäublin M.: Vigilance der Tierarzneimittel: Gemeldete unerwünschte Wirkungen im Jahr 2012. Schweiz. Arch. Tierheilk. 2013, 155: 613-620.

Müntener C., Kupper J., Naegeli H., Gassner B.: Vigilance der Tierarzneimittel: Gemeldete unerwünschte Wirkungen im Jahr 2015. Schweiz. Arch. Tierheilk. 2016, 158: 743-747.

Palm J., Von Krüger X., Ibrahim C.: Pharmakovigilanzreport Tierarzneimittel. Spontanmeldungen unerwünschter Arzneimittelwirkungen im Zeitraum 2011 bis 2013. Dtsch Tierärzteblatt. 2014, 2014 (11): 1540-1548.

Peacock RE., Hosgood G., Swindells KL., Smart L.: A randomized, controlled clinical trial of intravenous lipid emulsion

\section{Vigilanza dei medicamenti per uso veterinario: le notifiche degli effetti indesiderati nel 2016}

Nel 2016 sono state inoltrate 253 notifiche di effetti indesiderati di medicamenti per uso veterinario. Questo rappresenta rispetto allo scorso anno (292 notifiche), una diminuzione del 13\%. Principalmente sono state inoltrate notifiche relative a reazioni nei piccoli animali (178 cani e 32 gatti), nei bovini e vitelli (17 notifiche) e nei cavalli (10 notifiche). I gruppi di medicinali maggiormente messi in causa sono gli antiparassitari (145 notifiche), i preparati ormonali (26) e gli antiinfettivi (10). Al Tox Info Suisse sono stati trasmessi 32 casi, riguardanti principalmente l'ingestione in sovradosaggio di medicinali ad uso veterinario contenenti aromi e alcuni medicamenti con cambiamento di destinazione. Tra tutte le notifiche sono stati identificati 5 segnali che hanno portato ad un adeguamento delle informazioni del preparato in questione nelle rubriche "effetti indesiderati" oppure "controindicazioni".
Vigilance der Tierarzneimittel: Gemeldete unerwünschte Wirkungen im Jahr 2016

\section{C.R. Müntener et al.}

as an adjunctive treatment for permethrin toxicosis in cats. J. Vet. Emerg. Crit. Care (San Antonio). 2015, 25: 597-605.

Swissmedic: Merkblatt Erläuterungen zur Fachinformation, 2016. Zugänglich unter https://www.swissmedic.ch/ ZL000_00_017d_MB.

Tierarzneimittelkompendium der Schweiz: Hrsg. D. Demuth \& C. Müntener, Institut für Veterinärpharmakologie und -toxikologie der Universität Zürich, 2017. Zugänglich unter www.tierarzneimittel.ch.

Weber JCP.: Epidemiology of adverse reactions to nonsteroidal anti-inflammatory drugs. Adv. Inflam. Res. 1984,

6: 1-7.

\section{Korrespondenz}

Cedric R. Müntener

Institut für Veterinärpharmakologie und -toxikologie

Winterthurerstrasse 260

$\mathrm{CH}-8057$ Zürich

E-Mail: cedric.muentener@vetpharm.uzh.ch 